Fecha de recepción: mayo 2020 Fecha de aceptación: junio 2020

Versión final: julio 2020

\section{La otredad subyacente como creencia. Una reflexión ética, política y formativa en diseñadores de posgrado}

Leobardo Armando Ceja Bravo ${ }^{(*)}$

\begin{abstract}
Resumen: Reflexionar sobre el impacto ético, político en la formación de diseñadores resulta por demás importante. El objeto diseñado, comporta implicaciones de mediación, en cuyo efecto subyace el acontecimiento caracterizado por la afinidad, pero también por la confrontación, la ruptura o la emancipación de las personas. Estas implicaciones, no pueden pasar desapercibidas y constituyen un elemento fundamental de todo proceso de aprendizaje.

En el presente texto, se examinan y comparan algunas de las creencias subyacentes obtenidas por dos grupos de estudiantes de la Maestría en Gestión del Diseño Editorial de la Facultad de Diseño de la Universidad De La Salle, Bajío. Un primer grupo corresponde a estudiante del primer cuatrimestre, mientras que el segundo corresponde a estudiantes del quinto y último cuatrimestre de dicho posgrado. En ambos casos, se recurrió a la aplicación del test "Camino al Infierno" que Milton Glaser (2014) ha elaborado y que frecuentemente aplica a sus estudiantes. Los resultados obtenidos, sirven de telón de fondo para establecer un diálogo y reflexión a partir de la identificación narrativa descrita por los participantes, y ello dará cuenta del carácter complejo en el cual se encuentra inmersa la condición humana, puesto que la ética, la política y su práctica, tanto formativa como profesional, no pueden eludir con la responsabilidad cotidiana de su quehacer de diseñadores.
\end{abstract}

Palabras Clave: Ética - política - Otredad - creencia - diseño - complejidad

[Resúmenes en inglés y portugués en las páginas 184 - 185]

(*) Doctor en Desarrollo y Docencia del Diseño por parte de la Universidad Madero en Puebla. Obtuvo el Mérito Académico 2010-2011. Medalla Summa Cum Laude, otorgada por esa universidad. Es miembro del Sistema Nacional de Investigadores (SIN) nivel: candidato. Profesor Investigador de Tiempo Completo en la Facultad de Diseño de La Universidad De La Salle, Bajío. laceja@delasalle.edu.mx 


\section{Introducción}

El diseño es una actividad proyectual orientada a la resolución de problemas complejos en donde la otredad es el factor determinante y dador de pertinencia y posibilidad de dicho diseño. Asumir la existencia del otro, en tanto ser activo, implica la configuración de múltiples "representaciones de la sociedad" (Becker, 2015, p.22). Estas representaciones están mediadas por el objeto diseñado dentro de un contexto particular. Por tanto el objeto de diseño puesto en contexto requiere de marcos interpretativos base que sirvan de vínculo y entendimiento.

Orientar la reflexión sobre las implicaciones sociales del diseño, requiere entender esta actividad dentro de un contexto social, ello es, al mismo tiempo, hacerlo en términos políticos y éticos. Porque no se puede separar el carácter social y político del ético. Paul Ricoeur (2000) establece en Del texto a la acción. Ensayos de hermenéutica II, que las implicaciones éticas y políticas deben abordarse a la par de lo económico, puesto que al no hacerlo de esa forma, se corre el riesgo de aproximarse a dicha implicación en términos moralizantes, acción que resulta poco pertinente para aproximarse al presente tema. Desde la postura propuesta por Ricoeur (2000) es posible preguntarse sobre el momento en que la interpretación del objeto diseñado puede entenderse y explicarse como un texto, y el beneficio que puede tener plantearlo en estos términos, sabiendo que se puede dar un efecto en este "decir del objeto de diseño". Si se entiende al objeto de diseño, como un decir condensado, en tanto portador de significado, se requiere de la colaboración las personas, son ellas las que ponen en marcha la acción significativa del objeto diseñado, si esto es así, entonces es posible asociar al diseño con su designio, a saber, destino. (Flusser, 1999).

El ejercicio que sirve de telón de fondo en el presente texto, a saber, la aplicación de un pequeño test, elaborado expresamente por el diseñador norteamericano Milton Graser (2014), obliga a realizar una revisión sobre las implicaciones éticas de los diseñadores, ante supuestos que sólo tendrán sentido en la medida que el propio participante sea capaz de vincular dicho cuestionamiento con aspectos de su ejercicio profesional real. Este es uno de los criterios centrales que contribuye a la reflexión en el presente texto. Saber que se produce un efecto en el otro, que dentro del supuesto del cuestionario se encuentra ausente, pero que está vivo en la memoria del diseñador, en la medida en que éste se implica en el resultado de lo diseñado, resulta por demás interesante.

La mediación ejercida por los objetos de diseño, sean estos mensajes, objetos, espacios, entornos, ambientes o sistemas confieren una revisión, lectura e interpretación de los mismos, pudiendo entenderlos a partir de su lectura, es decir, se propone aproximarse a su estudio como texto, los cuales pueden ser leídos. Esta lectura del objeto de diseño, se constituye en posibilidad de entendimiento y verbalización por parte de las personas, configurando así su mundo, a partir de los juegos del lenguaje y formas de vida (Wittgenstein, 2012) imperante y subyacentes, a saber, “...delinean a su manera las delaciones entre el individuo y la colectividad." (Augé, 2014, p. 144) ahí es donde lo diseñado cobra otro sentido que el mero hecho de objeto configurado. 


\section{El diseño como un ejercicio de reconocimiento del otro.}

Contar con la identificación de un contexto social, en el que se inscribe el objeto de diseño puede establecer la existencia de un sin fin de circunstancias que en mayor o menor medida se pueden inscribir en lo diseñado. Entender el objeto de diseño dentro de un contexto social determinado, evidenciará una suma de circunstancias que, en conjunto dotan de sentido a dicho objeto, por ello, lo diseñado se entiende como un medio que posibilita el diálogo. En este sentido el objeto de diseño, debe contribuir a un diálogo, orientado hacia las personas y, en esta orientación descansa la acción, "una toma de decisión" (Olvera, 2013, p. 75) mediada por el vínculo entre las personas y el diseño.

El diseño, en el mejor de los casos, debería ser entendido como un ejercicio producto del reconocimiento del otro. En ese sentido, lo diseñado, es la voluntad del ejercicio dialógico, orientado a la acción reflexiva y prospectiva en el que “...el reconocimiento de otro cualquiera constituye un paso hacia el reconocimiento de todos” (Lévinas, 2001, p 108).

La consagración a la que el diseñador confiere sus esfuerzos queda transformada en las características que presenta el objeto de diseño, y puede orientarse a la búsqueda de ecos y resonancias que solo se pueden dar dentro del contexto social. En ese sentido, el objeto de diseño, al estar ante los demás, despliega las posibilidades discursivas inscritas en éste, por lo que "el discurso de lo que somos [a través del objeto de diseño] sólo da frutos ante los otros que callan para escucharnos." (Olvera, 2013, p. 100), pero uno de los inconvenientes que privan en el mundo actual, radica en la proliferación excesiva de objetos de diseño, todos en búsqueda de entablar un diálogo con las personas. El exceso de objetos de diseño, contribuye a la generación de discursos que sólo serán entendidos por los demás como sobreabundancia de información, transformándolo todo en lo mismo, lo idéntico, y ello implica unificación o como lo refiere Baudrillard (1991, p. 72) al afirmar que: "el que vive por lo mismo, perecerá por lo mismo."

El diseño, como actividad proyectual está presente en todos los aspectos de la vida social, al mismo tiempo, pareciera que ejerce un extraño efecto de desvanecerse, es decir, su valor y presencia cotidiana da por sentado su valor, existencia, utilidad y efecto unas veces positivo, otras negativo.

El diseño es portador de una lógica de integración, pero también de exclusión de las personas. No hay un diseño para todos, no se diseña para todos. De ahí su fuerza aglutinante pero también su poder de fragmentación.

El gran reto del diseño estará en re-ligar lo que se encuentra separado. Tramar el diseño, será pensarlo desde una postura política de reconocimiento del Otro, por medio de la cual sea capaz de contribuir al restablecimiento de lo social. Tramar el diseño será insertarlo en el contexto social, es propiciar, por tanto, una acción vinculante. De ahí, la importancia de reflexionar en ello.

Por su parte, Tim Ingold (2012) refiere que el trabajo de diseño es un asunto de temporalidad, es decir, gracias al diseño se logran resolver problemáticas acotadas a un tiempo y un espacio, pero al mismo tiempo, este mismo hecho puede interpretarse como un acto fallido. Dado que, con el paso del tiempo es necesaria la intervención de otro diseñador, para buscar la resolución de una problemática que ya había sido resuelta. Es en ese sentido en el 
que el antropólogo inglés afirma que: "Diseñar es darle forma al futuro del mundo en que vivimos. Sin embargo, por muchos motivos esto parece una empresa desesperanzadora fundada en los fracasos de nuestro predecesores." (Ingold, 2012, p. 19). Puede reinterpretarse la idea de fracaso a la luz del carácter dinámico y social en la cual se inscribe la propia actividad de diseñar, y con ello, subyace, el carácter complejo de la misma, transitorio, pero también, el reconocimiento, la participación y la fuerza social implicada.

En la actualidad, y con la proliferación de los medios digitales, la individualidad se acrecienta, pero al mismo tiempo, la idea de "colectividad" se hace evidente gracias al uso de la tecnología, exponencialmente los mensajes proliferan y se difunden, y con ello, también la idea de las “...multitudes inteligentes (smart mobs)..." (Han, 2016, p. 29).

El acceso digital conlleva la interacción ampliada de seres humanos, pero al mismo tiempo requiere de la particularización de dichas acciones. La paradoja se encuentra en el hecho de hacer evidente diversas problemáticas de comunicación y socialización, y en este sentido implican un espectro amplio de interacción e influencia. De esta forma, “....el homo digitalis se presenta con frecuencia de manera anónima, pero no es ningún nadie, sino que es un alguien, a saber, un alguien anónimo." (Han, 2016, p. 28).

En medio de este contexto de mediación digital, y en donde la interacción humana pareciera que se distancia físicamente, es importante preguntarse dónde está el diseño.

\section{¿Dónde está el diseño?}

El diseño es una acción humana que atraviesa todos los ámbitos de la vida. Al mismo tiempo, puede entenderse como la mediación de "ambientes que se requieren para sobrevivir". (Margolin 2017, p. 105). A modo explicativo y para valorar los aspectos éticos relacionados con el ejercicio profesional, en el presente artículo se analizará una encuesta administrada a los estudiantes de la Maestría en Gestión del Diseño Editorial de la Facultad de Diseño de la Universidad De La Salle Bajío. Dicha encuesta estaba integrada por 11 preguntas, las cuales requerían que los participantes contextualizaran su actividad profesional, en relación con diversas acciones de carácter ético. Parte de la lógica de esta encueta radica en que cada una de las preguntas resultará más comprometedora para los participantes, si su respuesta es afirmativa, puesto que las consecuencias de la decisión de diseño que toman, afecta la vida de los supuestos usuarios.

Algunos de los resultados que se obtuvieron con la aplicación de esta encuesta, y que de mejor forma pueden evidenciar la conciencia ética de los participantes, se refleja en los resultados obtenidos de las preguntas 4, 5, 7, 9 y 10 .

Ante la pregunta 4, ¿Estarías dispuest@ a...? Diseñar una portada de un libro cuyo contenido sexual encuentras personalmente repelente. Ocho de los veintiún participantes respondió que sí, ello equivale al 38.09\%. Doce de los participantes respondió que no, y ello equivale al $57.14 \%$. Solo un estudiante no contestó.

En relación con la pregunta 5, ¿Estarías dispuest@a...? Diseñar una campaña publicitaria para una empresa con un historial conocido de discriminación en materia de contrata- 
ción de minorías étnicas. Cinco de los participantes respondieron que sí, ello equivale al $23.80 \%$. Quince participantes respondieron que no, lo que equivale al $71.42 \%$. Solo un participante no respondió.

En relación con la pregunta 7, ¿Estarías dispuest@ a...? Diseñar una línea de camisetas para un fabricante que emplea mano de obra infantil. Dos de los participantes respondieron afirmativamente, y ello equivale al $9.52 \%$. Dieciocho respondieron que no, lo que equivale al $85.71 \%$. Solo un participante no contestó.

En relación con la pregunta 9, ¿Estarías dispuest@ a...? Diseñar un anuncio para un candidato político cuyas iniciativas sabes que serían dañinas para la población. Tres participantes respondieron que si, lo que equivale al $14.28 \%$. Diez y seis respondieron que no, lo que equivale a un $76.19 \%$. Solo un participante no contestó.

En relación con la pregunta 10, ¿Estarías dispuest@ a...? Diseñar un folleto promocional para un todoterreno que en condiciones de emergencia tiene una tendencia a volcar que supera la media y que ya ha causado la muerte de 150 personas. Cinco de los participantes respondieron afirmativamente, lo que equivale al $23.80 \%$. Quince respondieron que no, lo que equivale al $71.42 \%$. Solo un participante no contestó.

A partir del análisis arrojados por la encuesta se desprende que un elemento importante, radica en el nivel de conciencia que los participantes mostraron al responder el cuestionario. Con ello, es posible afirmar que el diseño es una actividad ética, evidenciada en el producto resultante. Es decir, en el propio objeto de diseño, en el que se plasman "mensajes, objetos, entornos, espacios, ambientes y sistemas complejos." (Buchanan, 1990, p.5). Al mismo tiempo, es posible reflexionar sobre el efecto que lo diseñado puede tener dentro de contexto sociales particulares, debido a la existencia de niveles comunicativos, simbólicos e interpretativos, con los cuales se consolidan procesos culturales.

Con la aplicación de esta pequeña encuesta, se buscó evidenciar el grado de conciencia de los participantes, así como el aporte que el diseño tiene dentro del contexto social. Otro aspecto que amerita una reflexión, está relacionado con el carácter prospectivo del diseño. El carácter proyectual, requiere que los diseñadores no dejen de lado el impacto que el diseño tiene en la vida de los demás. Diseñar implica buscar configurar el mundo de los otros. En ese sentido, las prácticas poco conscientes del diseño, estarían contraviniendo a esa tradición fundamental humana de descubrimiento, transformación y adaptación. Eso que Flusser (1999, p. 109) ha explicitado como “...el proceso gracias al cual la mano convierte progresivamente a la naturaleza en cultura.”. En tal sentido esta transformación atribuida a la disciplina que se está analizando está atravesada por valores, que se ponen de manifiesto en "...las interpretaciones y significados de los objetos diseñados..." (Heskett, 2005, p.47). Es decir que el significado asociado con todos los objetos de diseño, de una forma u otra expresan también “...los valores precisos que se les asignan...” (Heskett 2005, p 44). Por su parte, Francisco Irigoyen $(2008$, p. 16) entiende a la disciplina del diseño como “... una práctica fundamentalmente creativa cuyos mecanismos operatorios a nivel del pensamiento lo vinculan necesariamente a todos los campos del saber y el conocimiento.", en ese sentido el aporte que tiene el diseño, lo ubica como un nodo, en relación con el resto de las disciplinas, establece un diálogo profesional, en beneficio de las personas. 
Contar con este ejercicio, como evidencia del quehacer profesional y algunos de los supuestos éticos implicados en las respuestas vertidas por los participantes, es posible preguntarse de qué forma el diseño contribuye a una mejor comprensión del mundo que nos rodea.

\section{Cómo contribuye el diseño a una mejor comprensión de mundo}

Se ha comentado la implicación de mediación que comporta el objeto diseñado, al mismo tiempo, el lenguaje y la verbalización se lo hacen presente, gracias y sólo al vínculo que ejerce el objeto diseñado en la comprensión y aproximación de la realidad, sino al mundo que encarna en cada caso. En este tenor, es que el diseño también debe entenderse como un acontecimiento, en tanto posibilidad de transformar la realidad y el comportamiento de las personas. Del mismo modo, es necesario entender que: "Un "acontecimiento" puede hacer referencia a un desastre natural devastador o al escándalo más reciente provocado por una celebridad, al triunfo del pueblo o a un cambio político despiadado, a la intensa experiencia de una obra de arte o a una decisión íntima." (Zizek, 2014, p. 15).

El diseño, en tanto expresión subyacente de un acontecimiento deberá considerar, en todo momento, la complejidad humana y cómo ésta se relaciona con diversos aspectos implicados. Este ejercicio, tendrá como correlato, por tanto, el reconocimiento y ubicación espacial que el objeto diseñado tiene, y el modo en que dicha relación espacial es ejercida a través del tiempo, así como con los objetos configurados, actúan evidenciando diversos efectos sobre las personas.

En función de las distintas experiencias y expectativas relacionadas con las implicaciones del diseño, ésta será entendida por nociones que condicionen sus alcances. Pensar en un diseño desde la periferia, o como disciplina mediadora de saberes, contribuye a reflexionar en relación con otras disciplinas. Por el contrario, hacerlo desde una perspectiva centrada en la propia práctica del diseño, regresa al diseño a un punto en el que todo gira en torno a lo diseñado y ello reorienta la visión hacia la propia disciplina.

Existen para el ámbito del diseño dos retos fundamentales. El primero de ellos está en buscar trascender los límites a los que tradicionalmente se ha encuadrado el quehacer y la práctica del diseño. El segundo reto, está orientado hacia la permanencia y consolidación del carácter ético del diseño, los cuales deben preservarse en toda circunstancia.

Continuar en esta línea de respeto y ética del diseño es deseable a fin de pensar nuevas rutas que consideren aspectos relacionados con la sustentabilidad y sostenibilidad ligadas al diseño. Para lo cual sería importante generar procesos de "pérdida marginal 0" (Rifkin, 2015) el cual consiste en el cuidado a conciencia y puntual de los recursos que se requieren para la producción. Bajo este esfuerzo de producción se necesita considerar la cantidad de objetos o servicios que se pueden producir, evitando con ello una sobre necesidad de recursos.

Por tanto, las prácticas imperantes del diseño lo insertan en una lógica económica de producción lineal. Es decir, el diseño está al servicio de las demandas económicas, sin lograr incidir significativamente en materiales, procesos y objetos, en la medida en que las condiciones lo demandan. En algunos contextos, no se logran cubrir los parámetros de 
sustentabilidad y sostenibilidad que se requieren, ello además de poderse entender como una falta de responsabilidad, civismo y ética repercute significativamente con la idea o creencia que se tiene de las disciplinas proyectuales. Estas creencias erróneas sobre lo que dichas disciplinas son y lo que pueden aportar y contribuir socialmente, trasciende dichas ideas erróneas y con ello, revierten este efecto negativo, en función de lo que Víctor Margolin (2005, p. 127), expresa al afirmar que: "El diseño está inventando permanentemente su temática...", por lo que permanentemente debe revisar sus implicaciones, alcances e impactos sociales, y ello, ser una actividad ineludible para todos los implicados. En ese sentido, se sugiere la revisión de los siguientes seis conceptos.

\section{Conceptos que contribuyen a una comprensión del mundo.}

A continuación se presentan seis conceptos que se consideran fundamentales para una aproximación del mundo de hoy.

Primero: Resulta fundamental reflexionar sobre las implicaciones del objeto de diseño dentro del contexto social. Buscar las relaciones subyacentes, mostrará lo verdaderamente importante que se encuentra imbricado en la revisión del efecto que el objeto tiene para las personas. A saber, “...lo que importa es lo que resulta de la relación entre las cosas, del acoplamiento entre las cosas." (Tavares, 2008, p. 10). En este sentido puede afirmarse que los objetos diseñados, han contribuido de una forma u otra en el reconocimiento del mundo circundante. De allí que constituyan pautas o marcas que contribuyen a la orientación de las personas. Los objetos diseñados contribuyen a un estar en el mundo que, a través de los tiempos, se ha configurado para darle sentido y permanencia al ser humano.

Segundo: Siguiendo una afirmación de Víctor Margolin (2017, p. 100) que reza:

Los diseñadores tienen que entrar en la sociedad civil con toda su energía y buscar agresivamente socios que compartan su deseo de sanar el mundo. Entre los miles de socios y organizaciones existentes entre la sociedad civil son muchos los que podrían considerar bienvenida su colaboración....

Resulta fundamental que los diseñadores, piensen su quehacer, con un sentido más social $\mathrm{y}$ menos mercantil. Es fundamental entender el beneficio que representan los objetos diseñados para los diversos grupos o sectores que integran la sociedad. La labor debería estar orientada al respeto y reconocimiento de formas de bien vivir y, para ello, es importante considerar otros enfoques de diseño, como el diseño participativo.

\section{Tercero:}

Las disciplinas integradoras tienen como finalidad servir al enriquecimiento de la vida humana a través del entendimiento, la comunicación y la acción. El significado de buscar una base científica para el diseño no consiste en reducirlo 
a una o a otra de las ciencias, siendo una extensión del proyecto de los neopositivistas y así presentado en estos términos por algunos teóricos diseñadores. Más bien consiste en una conexión e integración del conocimiento útil de las artes y ciencias asociadas, pero, de forma que estén hechos en función a los problemas y propósitos del presente. (Buchanan, 1990, p. 2).

Asumir el potencial integrador dado por el diseño resulta ser un gran recurso. Tender puentes de saber y conocimiento disciplinar para, en conjunto dar respuesta a diversas problemáticas, es una de las características que posee el diseño. En ese sentido, será responsabilidad de los diseñadores tender los puentes necesarios para el diálogo, en beneficio de los más amplios y diversos sectores de la sociedad. Lo antedicho se articula fuertemente con el cuarto punto.

Cuarto: Flusser (1999, p, 164) afirma que: "Ser humano quiere decir ser un sistema complejo. Ser cuerpo quiere decir ser un sistema menos complejo. Ser cosas es una simplificación más del sistema..”

El pensamiento sistémico, es una condición necesaria en el diseñador. Las múltiples variables que están en juego en cada proyecto de diseño, hacen patente su carácter complejo. Lograr que el objeto de diseño sea, requiere concretar una serie de acciones que, en conjunto orientan y dotan de certidumbre al futuro.

Quinto: El diseño es una promesa de acción orientada al futuro, en ese sentido, el efecto que lo diseñado tendrá, debe considerar las implicaciones sociales de la misma. Para ello, es obligación del diseñador, entender el carácter profundamente humano, que subyace a toda intencionalidad de diseño, en la que el efecto que pueda tener, estará implicando a otros seres humanos, así como a las diversas formas de vida. Por tanto, resulta fundamental entender que

...el tema del diseño y los riesgos del cambio, primero abordando el tema de la complejidad de imaginar un futuro hacia el cual podrían dirigirse iniciativas de cambio significativo de la imagen y luego sobre cómo algunas de estas iniciativas podrían ser formuladas por la comunidad del diseño. (Margolin, 2017, p. 26).

Sexto: Los actos del diseño son acontecimientos-formas que subyacen en la realidad contextual. Por lo tanto, el diseño puede ser considerado una excelente herramienta para la búsqueda de una reconfiguración social. Pero para que ello ocurra, se precisa que las prácticas del diseño imperantes, sean seriamente cuestionadas para que "...los viejos modelos de la práctica” (Margolin, 2005, p. 140) que, dicho sea de paso, no están funcionando, sean modificados, de forma drástica y definitiva. 


\section{Reflexiones finales:}

El diseño se encuentra presente en todos y cada uno de los aspectos de la vida. Es portador de las más diversas ideas, valores e intenciones. Busca cumplir con la más amplia variedad de objetivos ligados a lo humano. En ese sentido, el diseño es el artificio cotidiano con el que es posible integrarse con los otros. Pero un mal diseño, podrá tener el efecto contrario a lo que se desea, el diseño en este sentido puede contribuir a la exclusión de las personas. El diseño es un concepto, y lo es por ser producto de una invención humana. Es un hecho histórico-cultural porque responde a los más profundos y sofisticados procesos creados por el ser humano.

En este sentido, no es aventurado afirmar que la cultura ha creado al diseño y éste se ha encarnado en la cultura. En esta misma línea de pensamiento, al objeto de diseño es posible entenderlo como un "artefacto cultural", es decir, un producto humano que posibilita la mediación inexorable en el contexto social.

Negar el aporte del diseño en su carácter mediador, requiere de una gran valentía, pero al mismo tiempo, exhibe una falta de conciencia y compromiso social. Es preciso pensarlo desde sus implicaciones político-sociales y, en este ejercicio, se encuentra inmersa la reflexión que el propio diseñador hace. Es el propio diseñador el responsable de las decisiones que toma en cada proyecto que lleva a cabo. Tramar el acontecimiento del diseño es prestar atención al efecto que el objeto diseñado tiene.

El gran reto del diseño estará centrándose en el re-ligar lo que se encuentra separado, en integrar lo que se encuentra disperso, en suponer la reducción de las contradicciones que su propio efecto entrópico ejerce.

Tramar el diseño será pensarlo desde una postura política de reconocimiento del otro, por medio del cual sea capaz de contribuir al restablecimiento de lo social, significando para las personas la posibilidad de integrarse como un actor comunicativo.

Para que el valor y aporte del objeto de diseño sea dado, se requiere del accionar de las personas, es decir, el ejercicio implicado de la otredad puesta en relación y diálogo a través del diseño. Cada vez que el diseñador toma conciencia de la existencia de otras personas, dejando de lado su condición de usuario, eleva la relación humana en términos éticos. Asumir un compromiso ético, implica considerar tanto a las personas como el tipo de materiales, procesos y requerimientos de producción cuyo efecto sea vinculante. Afrontar los retos de la actualidad en estos términos, resulta una invitación reflexiva para que los propios diseñadores revisen los modos, formas y maneras en las que se desarrollan los procesos del diseño. Todas estas acciones, deberán tener presente la necesidad ética centrada en un mejor, ser, hacer y relacionarse con los demás.

\section{Lista de Referencias Bibliográficas}

Augé, M. (2014) El antropólogo y el mundo global, México: Ed. Siglo XXI. Baudrillard, J. (1991) La transparencia del mal. Un ensayo sobre los fenómenos extremos, Barcelona: Ed. Anagrama. 
Becker, H. Para hablar de la sociedad la sociología no basta, Buenos Aires, Argentina: Ed. Siglo XXI.

Buchanan, R. (1990) Problemas Perversos en el Pensamiento del diseño, documento presentado en "Colloque Recherche sur le Design: Incitations, Implications, Interactions.", París, Francia, Universidad Tecnológica de Compiègne, Compiègne, Francia.

Flusser, V. (1999) Filosofía del diseño, la forma de las cosas, España: Ed. Síntesis.

Glaser, M. (2014) Diseñador / Ciudadano. Cuatro lecciones breves (más o menos sobre diseño), Barcelona, España: Ed. Gustavo Gili.

Han B. (2016) En el enjambre, Barcelona, España: Ed. Herder.

Hesket, Jonh, (2005) El diseño en la vida cotidiana, España: Ed, Gustavo Gili.

Ingold, T. (2012) Ambientes para la vida. Conversaciones sobre humanidad, conocimiento y antropología, Montevideo, Uruguay, Ed. Trilce.

Irigoyen, F. (2008) Filosofía y diseño: una aproximación epistemológica, México, Ed. UAMXochimilco.

Lévinas, E. (1991) Entre nosotros. Ensayos para pensar en otro, España: Ed. Pre-textos.

Margolin, V. (2005) Las politicas de lo artificial. Ensayos y estudios sobre diseño, México: Ed. Designio.

Margolin, V. (2017) Construir un mundo mejor. Diseño y responsabilidad social, México, Ed. Designio.

Olvera, L. (2013) La fragilidad del campamento. Un ensayo sobre el papel de la tolerancia, México: Ed. Almadía.

Rifkin, J. (2015) La sociedad de costo marginal cero, México: Ed. Paidós.

Tavares, G. (2008) Arquitectura, naturaleza y amor, en Opúsculo 14, julio 2008, Portugal, Dafne editora.

Wittgenstein, L. (2012) Tractatus logico-philosophicus, Madrid, España: Ed. Alianza Editorial. Zizek, S. (2014) Acontecimiento, España, Ed. Sexto Piso.

Abstract: Reflecting on the ethical and political impact on the training of designers is also important. The designed object carries mediation implications, in whose effect the event characterized by affinity, but also by the confrontation, rupture or emancipation of people underlies. These implications cannot go unnoticed and constitute a fundamental element of any learning process. In the present text, some of the underlying beliefs obtained by two groups of students of the Master in Editorial Design Management of the Faculty of Design of the De La Salle University, Bajío are examined and compared. A first group corresponds to a student of the first semester, while the second corresponds to students of the fifth and last semester of said postgraduate course. In both cases, the application of the "Road to Hell" test that Milton Glaser (2014) has developed and that frequently applies to his students was used.

The results obtained serve as a backdrop to establish dialogue and reflection based on the narrative identification described by the participants, and this will account for the complex nature in which the human condition is immersed, since ethics, politics and their 
practice, both formative and professional, cannot escape the daily responsibility of their designer work.

Keywords: Ethics - politics - Otherness - belief - design - complexity

Resumo: Também é importante refletir sobre o impacto ético e político na formação de designers. O objeto projetado carrega implicações de mediação, em cujo efeito o evento é caracterizado por afinidade, mas também pelo confronto, ruptura ou emancipação das pessoas subjacentes. Essas implicações não podem passar despercebidas e constituem um elemento fundamental de qualquer processo de aprendizagem.

Neste texto, examinamos e comparamos algumas das crenças subjacentes obtidas por dois grupos de estudantes do Mestrado em Gerenciamento de Design Editorial da Faculdade de Design da Universidade De La Salle, Bajío. Um primeiro grupo corresponde a um aluno do primeiro semestre, enquanto o segundo corresponde a alunos do quinto e último semestre do referido curso de pós-graduação. Em ambos os casos, foi usada a aplicação do teste "Road to Hell" que Milton Glaser (2014) desenvolveu e que se aplica frequentemente a seus alunos. Os resultados obtidos servem de pano de fundo para o estabelecimento de diálogo e reflexão a partir da identificação narrativa descrita pelos participantes, o que explicará a natureza complexa em que a condição humana está imersa, desde ética, política e sua prática, tanto formativa quanto profissional, não pode escapar da responsabilidade diária de seu trabalho de designer.

Palavras chave: ética - política - alteridade - crença - design - complexidade

[Las traducciones de los abstracts fueron supervisadas por el autor de cada artículo] 